\title{
28 Research Square \\ Landing Biomechanics During a Single-leg Landing With Lateral Trunk Obliquity in Female Athletes
}

\section{Shohei Taniguchi}

Hokkaido University

Tomoya Ishida ( $\nabla$ t.ishida@hs.hokudai.ac.jp)

Faculty of Health Sciences, Hokkaido University, Sapporo, Japan

\section{Masanori Yamanaka}

Hokkaido Chitose College of Rehabilitation

\section{Ryo Ueno}

Universität Innsbruck

Ryohei Ikuta

Hokkaido University

Masato Chijimatsu

Hokkaido University

Mina Samukawa

Hokkaido University

Yuta Koshino

Hokkaido University

\section{Satoshi Kasahara}

Hokkaido University

Harukazu Tohyama

Hokkaido University

\section{Research Article}

Keywords: Anterior cruciate ligament, Biomechanics, Prevention, Knee injury, Sex difference, Core stability, Knee valgus

Posted Date: January 28th, 2021

DOl: https://doi.org/10.21203/rs.3.rs-152869/v1

License: (9) (1) This work is licensed under a Creative Commons Attribution 4.0 International License. Read Full License 


\section{Abstract}

Background: Lateral trunk obliquity during landing is one of the characteristics of anterior cruciate ligament $(A C L)$ injuries in female athletes. Knee biomechanics during landing with lateral trunk obliquity may be different between female and male subjects. The purpose of the present study was to compare kinetics and kinematics in female subjects during landing with lateral trunk obliquity with those in male subjects.

Methods: Fifteen female (age $22.0 \pm 1.6$ years) and 15 male subjects (age $21.7 \pm 1.1$ years) participated in this study. The subjects performed a single-leg landing from a 30-cm-high box. Subjects were instructed to keep inclining their trunk to landing leg side at $15^{\circ}$ from vertical line. Kinetics and kinematics of their hip and knee joints were analyzed using a three-dimensional motion analysis system with a force plate and compared between female and male subjects.

Results: There were no significant differences in the angle of the laterally inclined trunk at initial contact (IC) and at the time of peak vertical ground reaction force (VGRF) $(P=0.341,0.363)$. The peak VGRF was smaller in female subjects than male subjects $(P=0.047)$. Hip adduction moment at the time of peak VGRF was significantly larger in female subjects than male subjects $(P=0.042)$. Hip external rotation of female subjects at IC was significantly larger than that of male subjects $(P=0.012)$. There were no differences in knee moments or kinematics between female and male subjects.

Conclusions: The findings of the present study indicated that female athletes are considered to avoid excessive knee abduction moment during landing with lateral trunk obliquity by kinematic and kinetic changes in their hip joint. Therefore, sports-medicine specialists should take more notice of kinematics and kinetic behaviors around hip joint in order to prevent noncontact ACL injuries for female athletes.

\section{Background}

Anterior cruciate ligament (ACL) injury is a severe sports injury. Approximately $70 \%$ of $A C L$ injuries occur in non-contact situations including cutting, pivoting or single-leg landing [1]. Female athletes have greater risk of ACL injury than male athletes [2]. The overall numbers of ACL injuries in female athletes remains higher than male athletes over a decade [2]. Knee abduction moment and abduction motion during a landing predicted primary and secondary ACL injuries [3, 4]. Large knee abduction moment cause high ACL strain as well as anterior tibial translation during cadaveric single-leg landing simulations [5-7]. Therefore, jump-landing training to prevent $A C L$ injury in female athletes have focused on reducing knee abduction moment [8]. However, female athletes do not always demonstrate larger knee abduction moment than male athletes during controlled landing task [9-12]. It is important to understand which task lead to gender-based differences in knee abduction moment since jump-landing training should be focused on landing tasks that derives large knee abduction moment in female athletes.

Lateral trunk obliquity toward a landing leg is considered to increase knee abduction moment by shifting center of mass [13-16]. Lateral trunk obliquity toward a landing leg increased knee abduction angle [17]. 
On the other hand, the landing instruction to keep trunk vertically in frontal plane decreased the peak knee abduction moment during a single-leg landing [18]. In addition, video analysis study showed that female athletes demonstrated lateral trunk obliquity toward a landing leg at ACL injuries, whereas male athletes did not [19]. Therefore, we hypothesized that the effect of lateral trunk obliquity on knee abduction moment in female subjects may be different from that of male subjects. Poor neuromuscular control of trunk was a significant predictor of ACL injury for female athletes but not for male athletes [20]. The purpose of the present study was to compare landing biomechanics during a single-leg landing with lateral trunk obliquity between female and male subjects.

\section{Methods}

\section{Subjects}

Thirty healthy subjects including 15 female subjects (age $22.0 \pm 1.6$ years, height $157.4 \pm 5.9 \mathrm{~cm}$, weight $52.4 \pm 4.9 \mathrm{~kg}$ ) and 15 male subjects (age $21.7 \pm 1.1$ years, height $172.9 \pm 5.2 \mathrm{~cm}$, weight $63.8 \pm 4.8 \mathrm{~kg}$ ) participated in the present study. All subjects had experiences with regular sports activities. Subjects were excluded from this study if they reported any history of musculoskeletal injuries within the previous 6 months. This study was performed in accordance with the Declaration of Helsinki. All subjects read and signed informed consent forms before their inclusion in this study. The present study was approved by the review board of our institute.

\section{Procedures and data correction}

Biomechanical data were collected from the right legs of all subjects. Static standing was recorded to perform scaling of each subject and to define zero-reference position. Then, subjects performed a singleleg landing task with leaning trunk laterally. Subjects stood with right leg on the 30-cm-high box and they inclined their trunk to right at $15^{\circ}$ (Fig. 1). The angle of the laterally inclined trunk was measured using standard plastic goniometer. The angle of laterally inclined trunk was decided based on the data of a previous study of video-analysis of ACL injuries [19]. They were ordered to drop off the box and landed with right leg on a force plate (Type 9286, Kistler AG, Winterthur, Switzerland) with keeping inclination of their trunk. During the landing task, subjects placed their hands on their iliac crests. Subjects were allowed to practice until they felt familiar with landing task. Three successive trials were recorded. A successive trial was defined as being able to stand still for at least 3 seconds after landing.

All data were recorded with Coretex 5.0.1 (Motion Analysis Corp., Santa Rosa, CA, USA) using a motion analysis system with six high-speed cameras (Hawk cameras, Motion Analysis Corp.) and the force plate. A total of 41 retroreflective markers were placed on the spinous process of $\mathrm{C} 7$ and Th10, sacrum, right iliac crest and medial knee, as well as left and right of shoulders, anterosuperior iliac spine, greater trochanter, hips, lateral knees, medial and lateral malleoli, heels, and second and fifth metatarsal heads, and cluster markers were placed on right thigh and shank [9]. Marker coordinate data and force data were sampled at $200 \mathrm{~Hz}$ and $1,000 \mathrm{~Hz}$. 


\section{Data processing and reduction}

The kinematics of knee and hip joint were calculated using a joint coordinate system with the SIMM 6.0.2 software (MusculoGraphics, Santa Rosa, CA, USA). Zero-references were set at knee and hip angles during the static standing trial. In addition, the angle of a laterally inclined trunk was calculated using a custom MATLAB program (MathWorks, Inc., Natick, MA, USA). The angle of a laterally inclined trunk was defined as being formed by the line between the markers of $\mathrm{C} 7$ and Th10 and the vertical line on frontal plane [21]. External moments of knee and hip joints were calculated using the inverse dynamics technique with the SIMM software. Initial contact (IC) to the ground was defined as when the vertical ground reaction force (VGRF) exceeded $10 \mathrm{~N}$ [22]. Knee and hip joint angles at IC were derived since the instruction was given to keep inclination of their trunk by IC. In addition, knee and hip joint angles and moments at the time of peak VGRF were derived because ACL injuries would occur at the time of peak VGRF [23]. Positive joint angles were indicated knee flexion, knee abduction, knee internal rotation, hip flexion, hip adduction, hip internal rotation and lateral trunk lean toward landing leg. Positive external moments were indicated knee flexion, knee abduction, knee internal rotation, hip flexion, hip adduction, hip internal rotation. All kinetics variables were normalized to subject's body weight.

\section{Statistical analysis}

Independent t-test was conducted to compare knee and hip kinetics and kinematics between female and male subjects using IBM SPSS Statistics 22 (IBM, Armonk, NY, USA). The statistical significance was set at $P<0.05$. In addition, Cohen's $d$ was calculated as an effect size. The Cohen's $d$ was interpreted as follows: $d>0.80$ large, $0.80>d>0.50$ medium, and $0.50>d>0.20$ small [24].

\section{Results}

The laterally inclined angle of the trunk at IC was $14.8^{\circ}$ for female subjects and $16.1^{\circ}$ for male subjects; that at the time of peak VGRF was $13.9^{\circ}$ for female subjects and $15.1^{\circ}$ for male subjects. There was no significant difference in the inclined angle at IC or at the time of peak VGRF between female and male subjects (at IC: $P=0.363, d=0.34$; at the time of peak VGRF: $P=0.341, d=0.35$ ) (Fig. 2). Female subjects demonstrated significantly smaller peak VGRF than male subjects with a medium effect size (female: $39.8 \pm 6.8 \mathrm{~N} / \mathrm{kg}$, male: $45.0 \pm 6.5 \mathrm{~N} / \mathrm{kg}, P=0.047, d=0.76$ ) (Fig. 3). Female subjects showed significantly larger hip adduction moment than male subjects with a medium effect size $(P=0.042, d=0.78)$ (Fig. 4a), while significant difference in knee abduction moment at the time of peak VGRF was not found between female and male subjects $(P=0.193, d=0.49)$ (Fig. $4 \mathrm{~b})$. There were no other significant differences in knee moments or hip moments at the time of peak VGRF (Table 1).

Female subjects landed with larger hip external rotation angle at IC than male subjects with a large effect size $(P=0.012, d=1.15)$ (Table 2). However, there were no other significant differences in knee joint angle or hip joint angle at IC or at the time of peak VGRF between female and male subjects (Table 2). 
Table 1

Comparison of hip and knee joint moments $(\mathrm{Nm} / \mathrm{kg})$ at peak vertical ground reaction force.

\begin{tabular}{|lllll|}
\hline & $\begin{array}{l}\text { Female } \\
(\mathbf{n}=15)\end{array}$ & $\begin{array}{l}\text { Male } \\
(\mathbf{n}=15)\end{array}$ & Pvalue & Cohen's $\boldsymbol{~}$ \\
\hline Hip flexion moment & $1.5(0.4)$ & $1.8(0.5)$ & 0.116 & 0.59 \\
\hline Hip adduction moment & $0.6(0.2)$ & $0.4(0.2)$ & 0.042 & 0.78 \\
\hline Hip internal rotation moment & $0.1(0.2)$ & $0.1(0.2)$ & 0.622 & 0.18 \\
\hline Knee flexion moment & $0.5(0.4)$ & $0.4(0.6)$ & 0.663 & 0.16 \\
\hline Knee abduction moment & $-0.1(0.2)$ & $-0.2(0.2)$ & 0.193 & 0.49 \\
\hline Knee internal rotation moment & $0.2(0.2)$ & $0.3(0.2)$ & 0.119 & 0.59 \\
\hline Mean (SD), Moments were normalized to body weight (Nm/kg). & \\
\hline
\end{tabular}


Table 2

Comparison of knee and hip joint angles (degree).

\begin{tabular}{|lllll|}
\hline & $\begin{array}{l}\text { Female } \\
(\mathbf{n = 1 5})\end{array}$ & $\begin{array}{l}\text { Male } \\
(\mathbf{n = 1 5})\end{array}$ & Pvalue & Cohen's $\boldsymbol{d}$ \\
\hline At initial contact & & & & \\
\hline Hip flexion angle & $17.0(4.0)$ & $16.4(4.9)$ & 0.743 & 0.12 \\
\hline Hip adduction angle & -12.3 & $-10.9(4.4)$ & 0.489 & 0.26 \\
\hline Hip internal rotation angle & $(5.7)$ & & & \\
\hline Knee flexion angle & $-5.6(3.2)$ & $-1.4(4.7)$ & 0.012 & 1.15 \\
\hline Knee abduction angle & $15.4(6.5)$ & $15.1(6.0)$ & 0.910 & 0.04 \\
\hline Knee internal rotation angle & $-0.5(1.9)$ & $-1.1(2.0)$ & 0.431 & 0.48 \\
\hline At the time of peak vertical ground reaction & $-2.5(4.1)$ & $-3.9(6.9)$ & 0.509 & 0.24 \\
force & & & & \\
\hline Hip flexion angle & $21.8(4.9)$ & $18.6(5.5)$ & 0.107 & 0.61 \\
\hline Hip adduction angle & $-8.6(6.5)$ & $-10.0(6.2)$ & 0.566 & 0.21 \\
\hline Hip internal rotation angle & $-3.6(4.6)$ & $-0.9(4.4)$ & 0.114 & 0.60 \\
\hline Knee flexion angle & $33.8(8.7)$ & $33.1(7.1)$ & 0.807 & 0.09 \\
\hline Knee abduction angle & $2.2(2.7)$ & $1.2(3.9)$ & 0.416 & 0.30 \\
\hline Knee internal rotation angle & $5.9(3.8)$ & $5.3(5.5)$ & 0.722 & 0.13 \\
\hline Mean (SD) & & & & \\
\hline
\end{tabular}

\section{Discussion}

The purpose of the present study was to investigate gender-based differences in knee and hip kinetics and kinematics during a single-leg landing with a lateral trunk obliquity. The results of this study showed that female subjects demonstrated larger hip adduction moment at the time of peak VGRF than male subjects whereas female subjects landed with smaller peak VGRF compared with male subjects. In addition, female subjects showed larger hip external rotation angle at IC than male subjects. However, we failed to find any gender-based differences in knee kinetics and kinematics including knee abduction moment.

In the present study, the angle of the laterally inclined trunk was set at $15^{\circ}$ before dropping off the box to investigate gender-based differences in knee and hip kinetics and kinematics during a landing with lateral trunk obliquity. Female and male subjects showed $14.8^{\circ}$ and $16.1^{\circ}$ of lateral trunk obliquity at IC and 
there were no gender-based differences in the angle of laterally inclined trunk at IC or peak VGRF. Therefore, the landing task with lateral trunk obliquity was properly conducted to compare knee and hip kinetics and kinematics between female and male subjects.

It was hypothesized that female subjects would show larger knee abduction angle and moment compared to male subjects during landing with lateral trunk obliquity. This was motivated by the fact that larger knee abduction angle and moment increased by the lateral trunk obliquity was a proposed female specific ACL injury mechanism $[13,17,25]$. However, we failed to find gender-based differences in knee kinetic or kinematics. Instead, female subjects showed larger hip external rotation angle at IC than male subjects as similar to previous studies [26, 27]. Since hip internal rotation is a part of dynamic knee valgus motion and associated with larger knee abduction moment [4, 28], increased hip external rotation would diminish dynamic knee valgus motion and knee abduction moment. Therefore, female subjects might attempt to reduce knee abduction moment and dynamic knee valgus motion by a increasing hip external rotation angle.

In the present study, female subjects showed larger hip adduction moment at the time of peak VGRF compared with male subjects. Larger hip adduction moment would be associated with higher activity of hip abductor muscles and smaller co-contraction with hip adductor muscles [13, 14, 29]. A recent study has shown that larger hip abductor force was associated with smaller knee abduction moment during landing [30]. Therefore, there is a possibility that female subjects might adapt to reduce knee abduction moment during landing with lateral trunk obliquity by increasing hip external rotation angle and hip adduction moment. Large VGRF is also one of the risk factors of ACL injury and it is associated with large knee abduction moment during a landing, especially with lateral trunk obliquity toward a landing leg $[3,13,23,30]$. Therefore, female subjects might adapt to reduce the peak VGRF during landing with lateral trunk obliquity by decrease in the peak VGRF as well as hip joint adaptations.

Concerning clinical relevance, the findings of the present study indicated that female athletes are considered to avoid excessive knee abduction moment during landing with lateral trunk obliquity by kinematic and kinetic changes in their hip joint. Therefore, sports-medicine specialists should take more notice of strength and motions around the hip joint in order to prevent noncontact ACL injuries for female athletes.

Some limitations should be acknowledged. First, the lateral trunk obliquity in the present study was intentional, and the landing was conducted in a well-controlled condition. In actual sports situations, the effect of lateral trunk obliquity on knee kinetics and kinematics may be different between female and male athletes. Second, we did not measure any muscle activities although we expected that the hip abductor activity would be larger in the female subjects than the male subjects based on the gender difference in the hip adduction moment. Future study should be conducted to evaluate gender differences in muscle activities to reveal the mechanism of hip adaptation. Third, the ACL strain or the load was not measured in the present study. In addition, it is unclear whether gender differences in hip kinetics and 
kinematics are related to the decrease or increase of the risk of ACL injury because the present study was a cross-sectional design.

\section{Conclusions}

Female subjects demonstrated larger hip adduction moment at the time of peak VGRF, larger hip external rotation angle at IC, and smaller peak VGRF during the landing with lateral trunk obliquity compared to male subjects, whereas there were no significant differences in knee kinetics and kinematics. These findings indicated that female athletes are considered to avoid excessive knee abduction moment during landing with lateral trunk obliquity by kinematic and kinetic changes in their hip joint.

\section{Abbreviations}

$\mathrm{ACL}$

anterior cruciate ligament; IC:initial contact; VGRF:vertical ground reaction force

\section{Declarations}

Ethics approval and consent to participate: This study was performed in accordance with the Declaration of Helsinki. All subjects read and signed informed consent forms before their inclusion in this study. This study was approved by the Hokkaido University Institutional Review Board (approval number: 15-81).

Consent for publication: Not applicable

Availability of data and materials: The data collected and analyzed in the present study are not publicly available due to ethical restrictions, but are available from the corresponding author on reasonable request.

Competing interests: The authors declare that they have no competing interests

Funding: The authors did not receive any grants for this study.

Authors' contributions: All authors made substantial contributions to the conception and design the work. ST, TI, RI and MC collected the data. ST, TI performed data processing. ST, TI, MY, RU, MS, YK, SK and HT organized the results and contributes on the manuscript discussion. ST, TI and HT drafted the manuscript or revised it. All authors read and approved the final manuscript.

Acknowledgments: Not applicable

\section{References}

1. Boden BP, Dean GS, Feagin JA, Garrett WE. Mechanisms of anterior cruciate ligament injury. Orthopedics. 2000;23:573-8. 
2. Agel J, Rockwood T, Klossner D. Collegiate ACL Injury Rates Across 15 Sports: National Collegiate Athletic Association Injury Surveillance System Data Update (2004-2005 Through 2012-2013). Clin J Sport Med. 2016;26:518-23.

3. Hewett TE, Myer GD, Ford KR, Heidt RS, Colosimo AJ, McLean SG, et al. Biomechanical measures of neuromuscular control and valgus loading of the knee predict anterior cruciate ligament injury risk in female athletes: A prospective study. Am J Sports Med. 2005;33:492-501.

4. Paterno M V., Schmitt LC, Ford KR, Rauh MJ, Myer GD, Huang B, et al. Biomechanical measures during landing and postural stability predict second anterior cruciate ligament injury after anterior cruciate ligament reconstruction and return to sport. Am J Sports Med. 2010;38:1968-78.

5. Bates NA, Schilaty ND, Nagelli C V., Krych AJ, Hewett TE. Multiplanar Loading of the Knee and Its Influence on Anterior Cruciate Ligament and Medial Collateral Ligament Strain During Simulated Landings and Noncontact Tears. Am J Sports Med. 2019;47:1844-53.

6. Ueno R, Navacchia A, Bates NA, Schilaty ND, Krych AJ, Hewett TE. Analysis of Internal Knee Forces Allows for the Prediction of Rupture Events in a Clinically Relevant Model of Anterior Cruciate Ligament Injuries. Orthop J Sport Med. 2020;8:2325967119893758.

7. Navacchia A, Bates NA, Schilaty ND, Krych AJ, Hewett TE. Knee Abduction and Internal Rotation Moments Increase ACL Force During Landing Through the Posterior Slope of the Tibia. J Orthop Res. 2019;37:1730-42.

8. Sugimoto D, Myer GD, Foss KDB, Hewett TE. Specific exercise effects of preventive neuromuscular training intervention on anterior cruciate ligament injury risk reduction in young females: metaanalysis and subgroup analysis. Br J Sports Med. 2015;49:282-9.

9. Ishida T, Koshino Y, Yamanaka M, Ueno R, Taniguchi S, Samukawa M, et al. The effects of a subsequent jump on the knee abduction angle during the early landing phase. BMC Musculoskelet Disord. 2018;19:379.

10. Kernozek TW, Torry MR, Iwasaki M. Gender differences in lower extremity landing mechanics caused by neuromuscular fatigue. Am J Sports Med. 2008;36:554-65.

11. Orishimo KF, Liederbach M, Kremenic IJ, Hagins M, Pappas E. Comparison of landing biomechanics between male and female dancers and athletes, part 1: Influence of sex on risk of anterior cruciate ligament injury. Am J Sports Med. 2014;42:1082-8.

12. Weinhandl JT, Joshi M, O'Connor KM. Gender comparisons between unilateral and bilateral landings. J Appl Biomech. 2010;26:444-53.

13. Hewett TE, Myer GD. The mechanistic connection between the trunk, hip, knee, and anterior cruciate ligament injury. Exerc Sport Sci Rev. 2011;39:161-6.

14. Powers CM. The Influence of Abnormal Hip Mechanics on Knee Injury: A Biomechanical Perspective. J Orthop Sports Phys Ther. 2010;40:42-51.

15. Jamison ST, Pan X, Chaudhari AMWW. Knee moments during run-to-cut maneuvers are associated with lateral trunk positioning. J Biomech. 2012;45:1881-5. 
16. Dempsey AR, Elliott BC, Munro BJ, Steele JR, Lloyd DG. Whole body kinematics and knee moments that occur during an overhead catch and landing task in sport. Clin Biomech. 2012;27:466-74.

17. Saito A, Okada K, Sasaki M, Wakasa M. Influence of the trunk position on knee kinematics during the single-leg landing: implications for injury prevention. Sport Biomech. 2020:1-14.

18. Chijimatsu M, Ishida $T$, Yamanaka $M$, Taniguchi $S$, Ueno $R$, lkuta $R$, et al. Landing Instructions Focused on Pelvic and Trunk Lateral Tilt Decrease the Knee Abduction Moment during a Single-leg Drop Vertical Jump. Phys Ther Sport. 2020;46:226-233.

19. Hewett TE, Torg JS, Boden BP. Video analysis of trunk and knee motion during non-contact anterior cruciate ligament injury in female athletes: lateral trunk and knee abduction motion are combined components of the injury mechanism. Br J Sports Med. 2009;43:417-22.

20. Zazulak BT, Hewett TE, Reeves NP, Goldberg B, Cholewicki J. Deficits in neuromuscular control of the trunk predict knee injury risk: A prospective biomechanical-epidemiologic study. Am J Sports Med. 2007;35:1123-30.

21. Davies JL, Button K, Sparkes V, van Deursen RW. Frontal plane movement of the pelvis and thorax during dynamic activities in individuals with and without anterior cruciate ligament injury. Knee. 2018;25:997-1008.

22. Ford KR, Myer GD, Hewett TE. Reliability of landing 3D motion analysis: implications for longitudinal analyses. Med Sci Sports Exerc. 2007;39:2021-8.

23. Koga H, Nakamae A, Shima Y, Iwasa J, Myklebust G, Engebretsen L, et al. Mechanisms for noncontact anterior cruciate ligament injuries: Knee joint kinematics in 10 injury situations from female team handball and basketball. Am J Sports Med. 2010;38:2218-25.

24. Cohen J. Statistical Power Analysis for the Behavioral Sciences. 2nd ed. Abingdon(GB): Routledge; 1988.

25. Quatman CE, Hewett TE. The anterior cruciate ligament injury controversy: Is "valgus collapse" a sexspecific mechanism? Br J Sports Med. 2009;43:328-35.

26. Scattone Silva R, Serrão FV. Sex differences in trunk, pelvis, hip and knee kinematics and eccentric hip torque in adolescents. Clin Biomech (Bristol, Avon). 2014;29:1063-9.

27. Weltin E, Gollhofer A, Mornieux G. Effect of gender on trunk and pelvis control during lateral movements with perturbed landing. Eur J Sport Sci. 2016;16:182-9.

28. McLean SG, Huang X, van den Bogert AJ. Association between lower extremity posture at contact and peak knee valgus moment during sidestepping: implications for ACL injury. Clin Biomech (Bristol, Avon). 2005;20:863-70.

29. Patrek MF, Kernozek TW, Willson JD, Wright GA, Doberstein ST. Hip-abductor fatigue and single-leg landing mechanics in women athletes. J Athl Train. 2011;46:31-42.

30. Ueno R, Navacchia A, DiCesare CA, Ford KR, Myer GD, Ishida T, et al. Knee abduction moment is predicted by lower gluteus medius force and larger vertical and lateral ground reaction forces during drop vertical jump in female athletes. J Biomech. 2020;103:109669. 


\section{Figures}

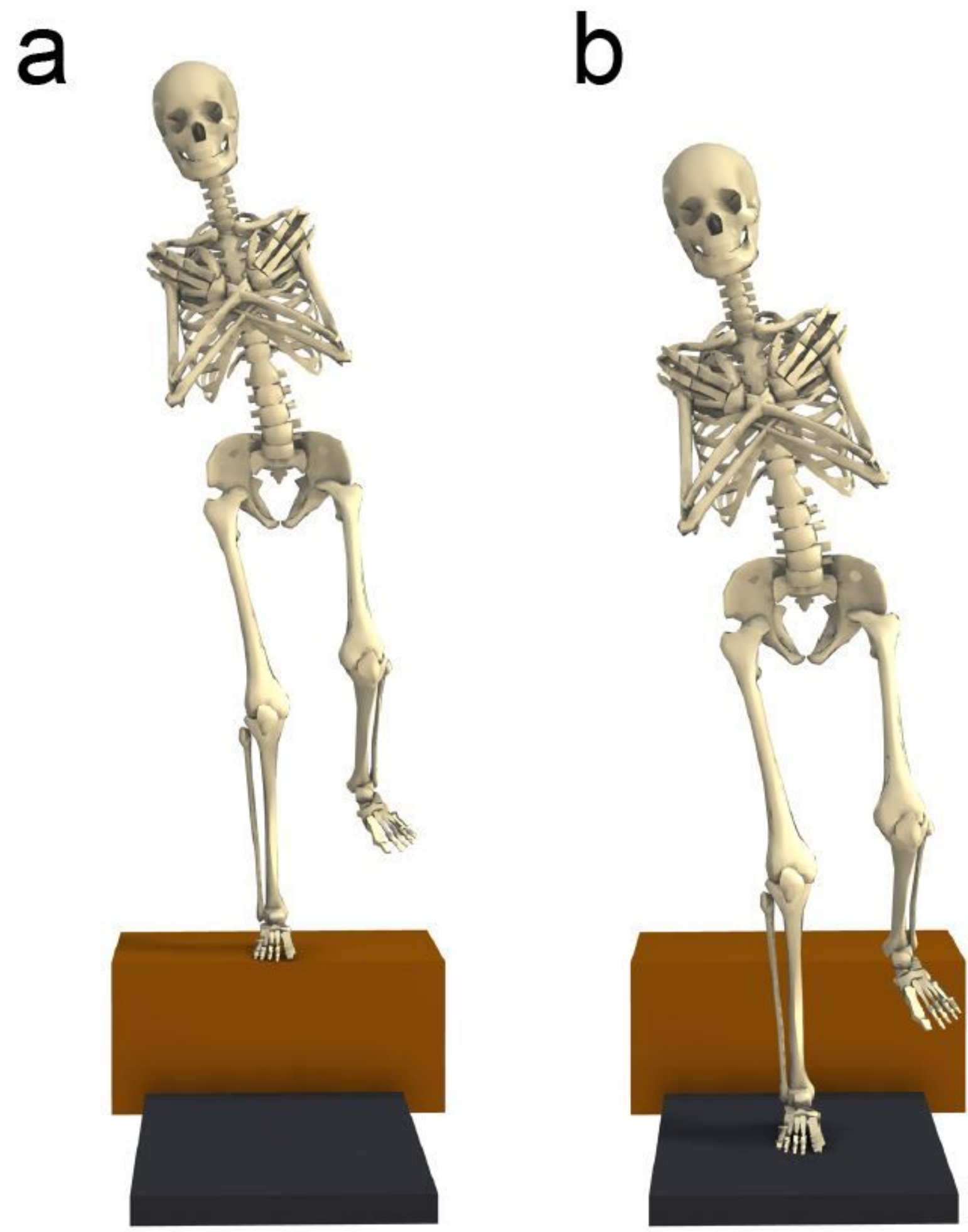

\section{Figure 1}

Drop landing task Subjects stand on a box and incline their trunk toward a landing leg at $15^{\circ}(\mathrm{a})$ and then land on a force plate with same side of landing leg and with keeping lateral trunk obliquity (b). 


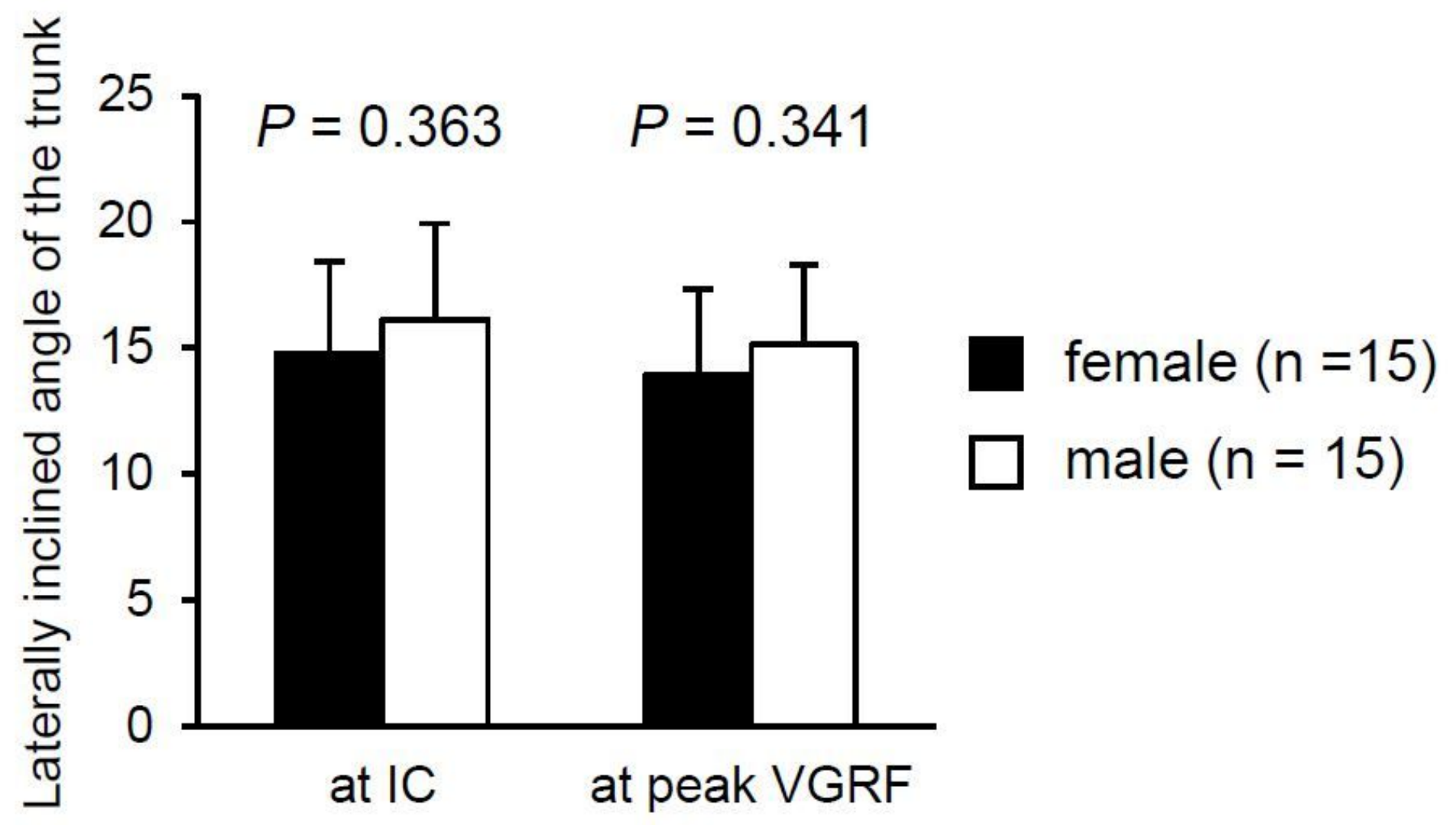

Figure 2

Comparison of laterally inclined angle of the trunk toward a landing leg. IC: initial contact; VGRF: vertical ground reaction force 


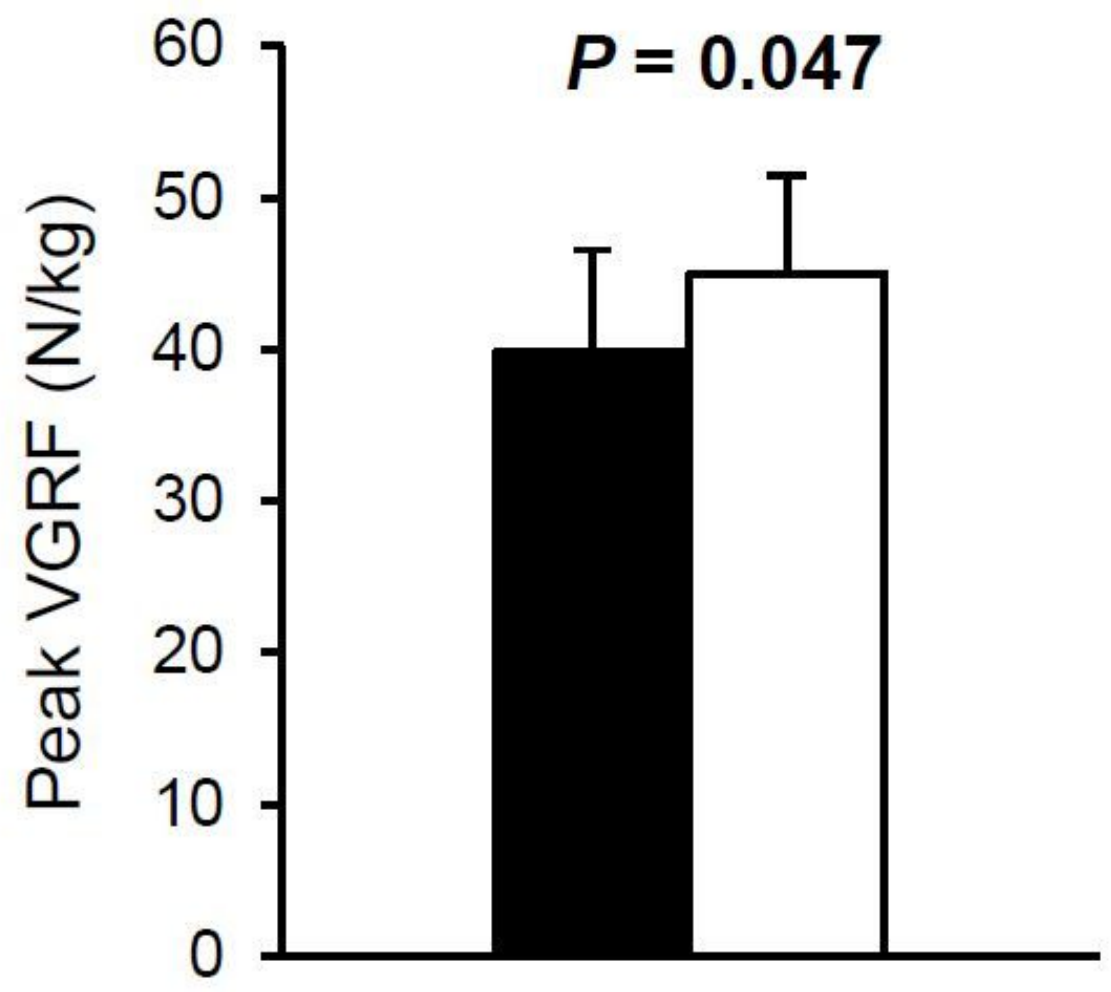

female $(\mathrm{n}=15)$

$\square$ male $(\mathrm{n}=15)$

Figure 3

Comparison of peak vertical ground reaction force (VGRF) between female and male subjects. VGRF was normalized to body weight.
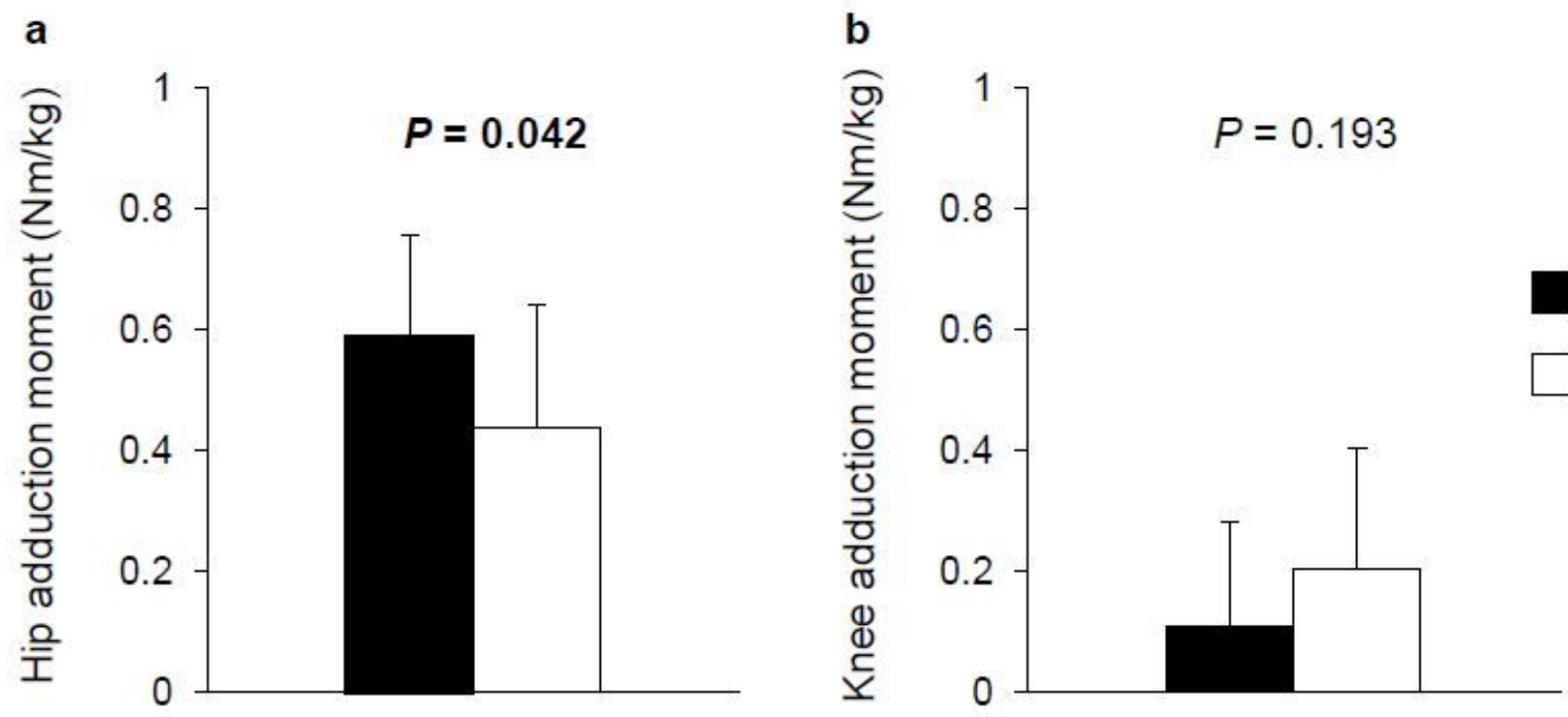

Figure 4

Comparison of external hip adduction and knee abduction moments at peak vertical ground reaction force. Moments were normalized to body weight. 